\title{
iTRAQ-based quantitative proteomics reveals a ferroptosis-like programmed cell death in plants infected by a highly virulent tobacco mosaic virus mutant 24A+UPD
}

\author{
Mercy Macharia', Prem P. Das ${ }^{1}$, Naweed I. Naqvi ${ }^{1,2^{*}}$ and Sek-Man Wong ${ }^{1,2,3^{*}}$ (i)
}

\begin{abstract}
Plants trigger a highly orchestrated defence mechanism in response to viral infection. In this study, we aimed at understanding the molecular events that lead to more accelerated cell death in Nicotiana benthamiana plants infected with the fast-replicating TMV 24A + UPD in comparison to TMV. TMV 24A + UPD is an artificial mutant that induces more severe symptoms leading to precocious death in plants. We employed the iTRAQ-based quantitative proteomics approach to identify and map the proteomes of TMV and TMV 24A + UPD infected plants at time points that correlate with initiation of early cell death symptoms. TMV 24A + UPD proteome profile revealed 183 highly abundant proteins versus 71 for TMV infected plants. KEGG analysis revealed differentially abundant proteins in the two proteome profiles under cell death, stress signalling, protein folding, sorting, degradation, transport and catabolism. We identified unique differentially abundant proteins in the TMV 24A + UPD profile, in particular under the ferroptosis and glutathione metabolism pathways. For validation, we varied the amount of intracellular iron by supplementing plants with $\mathrm{Fe}^{3+}$, employing iron chelators and by virus induced gene silencing of iron storage protein ferritin gene. We also employed potent ferroptosis inhibitors ferostatin-1, liprostatin-1, and transiently silenced glutathione peroxidase 4 gene. TMV $24 \mathrm{~A}+$ UPD infected plants showed accelerated cell death symptoms when intracellular iron was increased. Decreasing intracellular iron protected the plants from accelerated cell death. We also observed a decrease in TMV 24A + UPD induced cell death when we applied ferroptosis inhibitors. Glutathione peroxidase 4 gene-silenced plants showed enhanced cell death compared to non-silenced control plants. Our study uncovered a link between intracellular iron and accelerated lipid ROS-induced cell death in TMV 24A + UPD infected plants. We propose that the fast-replicating mutant of TMV induces a distinct and potent form of cell death akin to ferroptosis.
\end{abstract}

Keywords: iTRAQ, TMV, TMV 24A + UPD, Cell death, Glutathione peroxidase, Intracellular iron, Ferritin, Ferroptosis

\section{Background}

Plant viruses have a significant impact on plant production. In response to viruses, plants mount intricate protection systems that include crosstalk between many cellular pathways and immune response. Viruses depend on host cells for successful establishment (Wang and Li 2012). The interplay

\footnotetext{
* Correspondence: naweed@tll.org.sg; dbswsm@nus.edu.sg

${ }^{1}$ Department of Biological Sciences, National University of Singapore,

Singapore 119543, Singapore

Full list of author information is available at the end of the article
}

between virus and host factors continues throughout the lifecycle of the virus and has an impact on pathogenesis.

Such interaction with viruses perturbs the molecular networks in plant cells and triggers expression of hundreds of genes involved in defense, susceptibility and resistance (Di Carli et al. 2012; Das et al. 2018; GarciaRuiz 2018). One such plant defence responses is regulated cell death, whereby damaged or unwanted cells are eliminated through specific biochemical pathways (Reape et al. 2008). Cell death in mammals is well characterized into apoptotic or non-apoptotic pathways

(c) The Author(s). 2020 Open Access This article is distributed under the terms of the Creative Commons Attribution 4.0 International License (http://creativecommons.org/licenses/by/4.0/) which permits unrestricted use, distribution, and 
(Elmore 2007). Although plant and animal immune systems share similarities of events leading to cell death, details in plants are less known (Mukhtar et al. 2016).

The genomes of most tobamoviruses including the Tobacco mosaic virus (TMV) have a 3' untranslated region (UTR) that consists of an upstream pseudoknot domain (UPD) that plays an important role in translation (Niu et al. 2015). A mutated TMV was constructed by introducing different lengths of internal poly (A) tracts upstream of the UPD (Guo et al. 2015). One such mutant, TMV 24A + UPD, induced more severe leaf necrosis and stem collapse, leading to plant death within a few days. The difference in rate of virus replication and plant death are likely resulted from interactions between the introduced poly (A) tract, UPD and host factors. Host protein elongation factor 1A has been reported to interact with UPD of 3' UTR (Zeenko et al. 2002). The pathways involved in the accelerated cell death by TMV 24A + UPD have not been identified.

Thus, to understand TMV 24A + UPD interaction with the host and events leading to the altered pathogenesis, we compared the proteome profiles of TMV 24A + UPD or TMV-infected plants using the Isobaric Tags for Relative and Absolute Quantitation (iTRAQ) method. We compared the proteome profiles of TMV- or TMV 24A + UPD inoculated $N$. benthamiana plants at two different time points ( 2 and 3 days post inoculation, dpi) that correlate with the first signs of virus-induced cell death.

Interestingly, the KEGG (Kyoto Encyclopedia of Genes and Genomes) analysis revealed differentially abundant proteins (DAPs) unique to glutathione metabolism and ferroptosis pathways in TMV 24A + UPD infected plants. We identified a cytosolic iron chaperone poly $(\mathrm{rC})$-binding protein 1 (PCBP1) which delivers iron to ferritin. Ferritin is known to store iron in a non-toxic state (Leidgens et al. 2013). We also identified the antioxidant glutathione peroxidase. Ferroptosis is a regulated form of cell death driven by accumulation of lipid based reactive oxygen species (ROS) (Dixon et al. 2012). This cell death is dependent on free intracellular iron, which is required for the accumulation of toxic lipid ROS (Yang et al. 2014). We also identified glutathione peroxidase. In mammalian systems, glutathione peroxidase 4 (GPX4) is a key regulator of ferroptosis (Maiorino et al. 2018; Seibt et al. 2019). Based on the iTRAQ results, we infer that intracellular iron plays a key role in TMV 24A + UPD-ROS induced cell death. Further, our results demonstrated the link between intracellular iron and accelerated ROS induced cell death in TMV 24A+ UPD infected plants.

\section{Results}

\section{Protein identification and quantification}

There are various host factors involved in activation of immune response during early stages of virus infection (Lietzen et al. 2011). We employed high throughput proteomics to identify host factors that contribute to the rapid cell death induced by TMV 24A + UPD in comparison to TMV in $N$. benthamiana plants. We confirmed infection before sample processing, TMV coat protein was detected at 2 and $3 \mathrm{dpi}$ in both TMV and TMV 24A + UPD inoculated plants. For each time point, four biological replicates were included in the samples and grouped into two for iTRAQ tagging (Fig. 1).

Overall, proteins identified covered a wide range of biological processes. TMV 24A + UPD induced a significantly higher number of highly abundant proteins (HAPs) compared to that of TMV (Fig. 2a). Of the HAPs, 17 overlapped between TMV and TMV 24A + UPD. For less abundant proteins (LAPs), 56 overlapped between the two viruses (Fig. 2a, b).

Approximately 77,354 MS spectra identified matched the known spectra. Overall, a total of 15,996 peptides and 2166 proteins were identified (Fig. 2c). A total of 823 proteins showed differential accumulation, 354 were classified as HAPs and 469 as LAPs (Fig. 2c).

To confirm the iTRAQ quantification results, qRTPCR was performed for 21 selected genes encoding differentially abundant proteins of interest from TMV and TMV 24A + UPD infected plants. Some genes including hypersensitive-induced response, peroxiredoxin-2e-2, and programmed cell death proteins were selected based on their involvement in plant defense and cell death. Chloroplast associated genes were selected based on their low abundance in both proteome profiles. From the TMV infected plants, 5 of the selected genes were up-regulated, while 5 were down-regulated (Additional file 1: Figure S1a). From the TMV 24A + UPD infected plants, 8 genes were up-regulated while 3 were down-regulated (Additional file 1: Figure S1b). From the qRT-PCR results, 19 of the selected genes were upregulated or down-regulated as expected, thus validating our iTRAQ results. Taken together, we conclude that $N$. benthamiana proteome is significantly altered upon TMV and TMV 24A + UPD infection.

\section{Gene ontology (GO) enrichment and KEGG analysis of differentially abundant proteins}

Proteomic data of identified and quantified proteins were further analysed using different bioinformatics tools. The total differentially expressed genes in TMV and TMV $24 \mathrm{~A}+$ UPD infected plants were $13 \%$ vs $15 \%$ for biological processes, $56 \%$ vs $58 \%$ for cellular processes respectively. Molecular function was $29 \%$ for both profiles. When highly abundant proteins were analyzed separately, TMV $24 \mathrm{~A}+$ UPD revealed more GO terms including sulphur metabolism, immune system process, golgi membranes, plant vacuole, proteasome and protein phosphatase type 2A (Fig. 3a, b). Some terms were common in both profiles, namely ubiquitin ligase complex and ROS. The term Pbody was only found in TMV profile (Fig. 3a). 

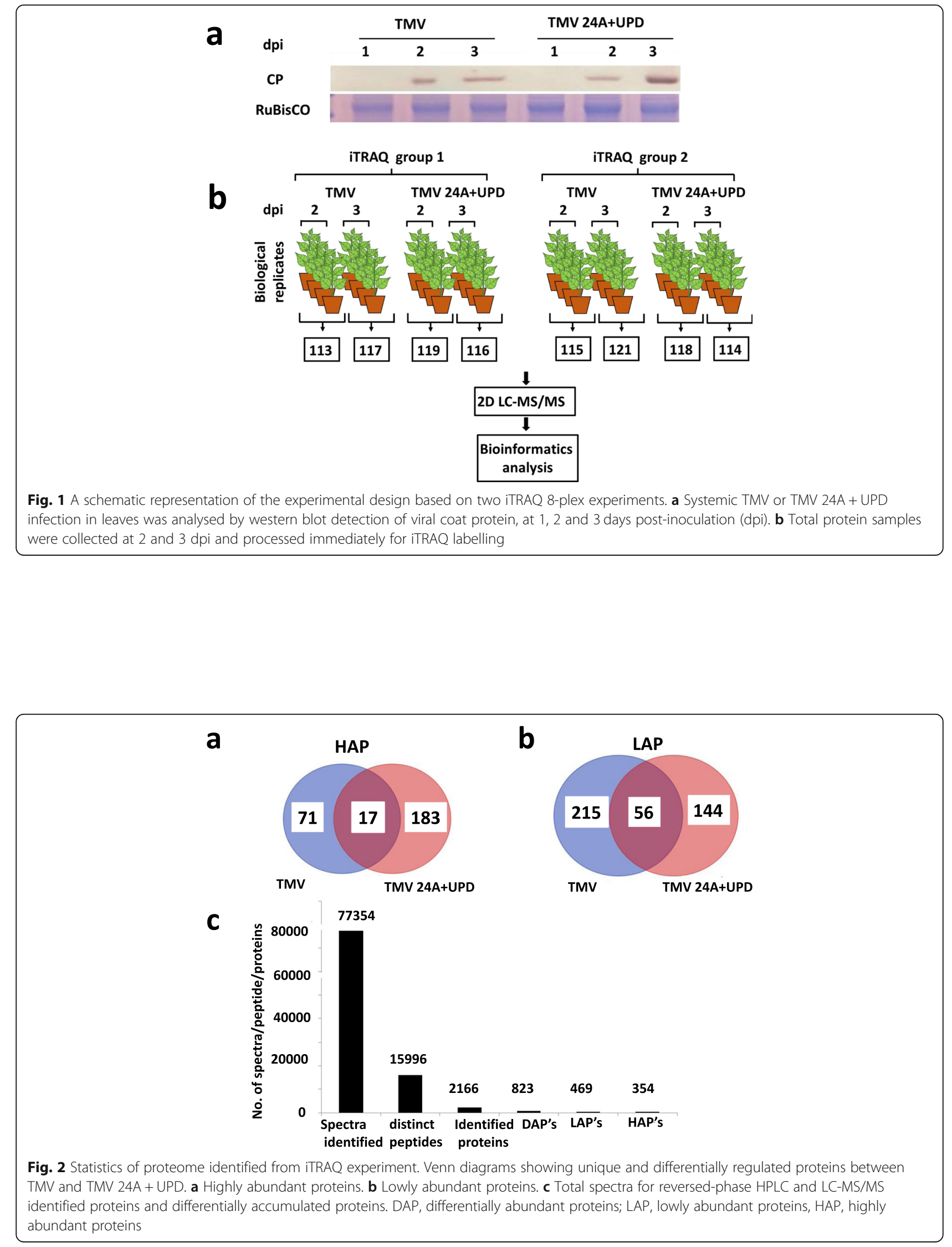


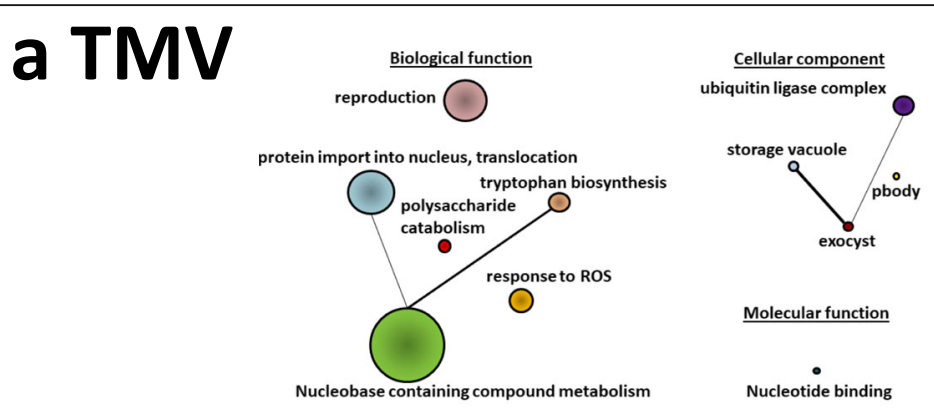

\section{b TMV 24A+UPD}

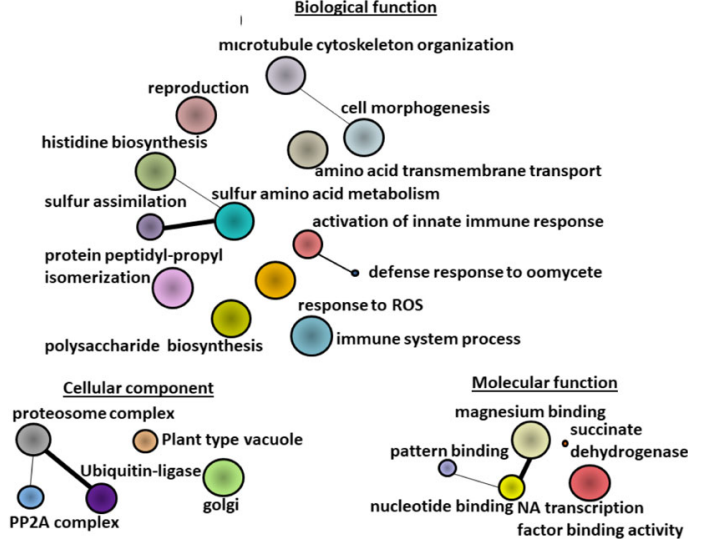

Fig. 3 Gene ontology (GO) enrichment analysis of differentially expressed proteins (DAPs) by TMV and TMV 24A + UPD infection. Panels showing interactive graphs of DAPs in TMV and TMV 24A + UPD infection. a High-abundant differentially expressed proteins in TMV proteome profile. $\mathbf{b}$ Highly abundant proteins in TMV 24A + UPD. The sizes of the circles are proportional to the number of proteins associated with the specific term. The interactive network was summarized and plotted following the published REVIGO protocol (http://revigo.irb.hr)

From the plant defence perspectives, 4 main functional groups were analysed (Fig. 4a, b), as classified by KEGG. There were differences in DAPs between TMV and TMV 24A + UPD associated with cellular senescence, apoptosis, necroptosis, glutathione metabolism and ferroptosis (Additional file 2: Table S1). Autophagy proteins were highly abundant with TMV 24A + UPD, while signalling pathways proteins involved in mTOR, PI3KAkt and sphingolipid signalling pathways were highly abundant in TMV proteome profile (Fig. 4a, b). MAPK signalling pathway proteins were more abundant in TMV 24A + UPD but less so with TMV. SNARE proteins involved in vesicle fusion were highly abundant with TMV 24A + UPD but not with TMV (Fig. 4b). The difference in GO terms and proteins in selected pathways between the two viruses suggest that the internal poly (A) tract in the UPD influences the pattern of plant-pathogen interaction. We conclude that the accelerated cell death in TMV 24A + UPD infected plants is preceded by increased and distinct perturbation, as compared to TMV infection.
Validation of iron-induced oxidative stress in TMV 24A + UPD infected $N$. benthamiana plants

Our analysis revealed that in contrast to TMV, the TMV 24A + UPD mutant induced accumulation of glutathione peroxidase, and peroxiredoxin ROS regulators (Additional file 2: Table S1). Our data also revealed an intracellular iron regulator, PCBP1, in TMV 24A + UPD samples but not in TMV. We sought to investigate if there was a relationship between iron and oxidative stress induced by TMV 24A + UPD. We first investigated oxidative stress in virus infected plants using TBARS assay. TMV 24A + UPD showed a significantly higher amount of MDA content at 3 dpi compared to mock and TMV infected plants (Fig. 5a).

When plants supplemented with $\mathrm{Fe}^{3+}$ in the form of ethylenediaminetetraacetic acid ferric sodium salt were infected with virus, TMV 24A + UPD plants showed an accelerated cell death compared to the control plants (Fig. 5b). TMV 24A + UPD infected leaves showed more severe chlorotic blotches and collapsed quicker than the control plants (Fig. 5c). 


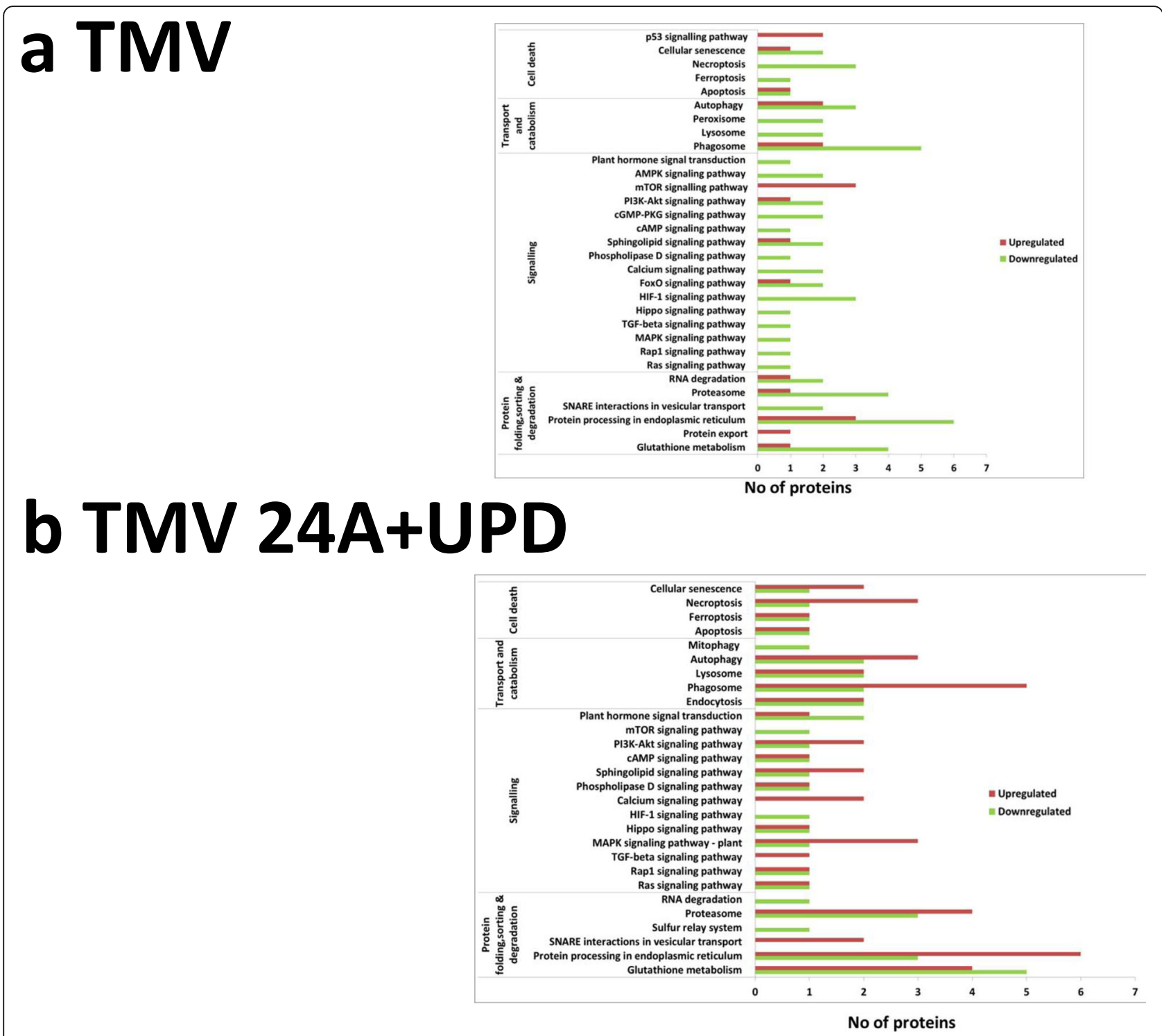

Fig. 4 KEGG pathway analysis for TMV and TMV 24A + UPD proteome profiles. Classification of the identified differentially accumulated proteins and their distribution in the KEGG pathways predicted for TMV (a) and TMV 24A + UPD (b) proteome profiles. Highly abundant proteins are shown as up-regulated while lowly abundant proteins are denoted down-regulated. X-axis represents number of proteins identified

After infiltrating the iron chelator deferoxamine mesylate (DFO) into the virus infected plants, cell death was significantly decelerated in TMV 24A + UPD inoculated plants but not in the mock or TMV inoculated plants (Fig. 6a, b). We also observed cell death inhibition with ciclopirox olamine (CPX)-treated TMV 24A + UPD inoculated plants but not in mock and TMV inoculated plants (Additional file 3: Figure S2). To further investigate the role of iron, we silenced ferritin gene by virus induced gene silencing (VIGS). TMV 24A + UPD infected plants exhibited slightly enhanced cell death symptoms compared to plants with non-silenced intact ferritin gene at $6 \mathrm{dpi}$ (Fig. 6c), the symptoms were enhanced more at 12 dpi (Additional file 4: Figure S3). We did not observe a significant difference of symptoms in ferritin-silenced and control plants when TMV was inoculated (Fig. 6c). These results suggest that intracellular iron accelerates accumulation of ROS in TMV 24A + UPD. We infer that a ferroptosis-like phenomenon forms part of the cell death response induced by TMV $24 \mathrm{~A}+$ UPD in N. benthamiana plants.

\section{Effect of ferroptosis inhibition in infected plants}

Ferrostatin-1 (Fer-1) and liprostatin-1 (Lip-1) inhibit ferroptosis via blocking the iron induced ROS accumulation, particularly lipid peroxidation (Skouta et al. 2014). 


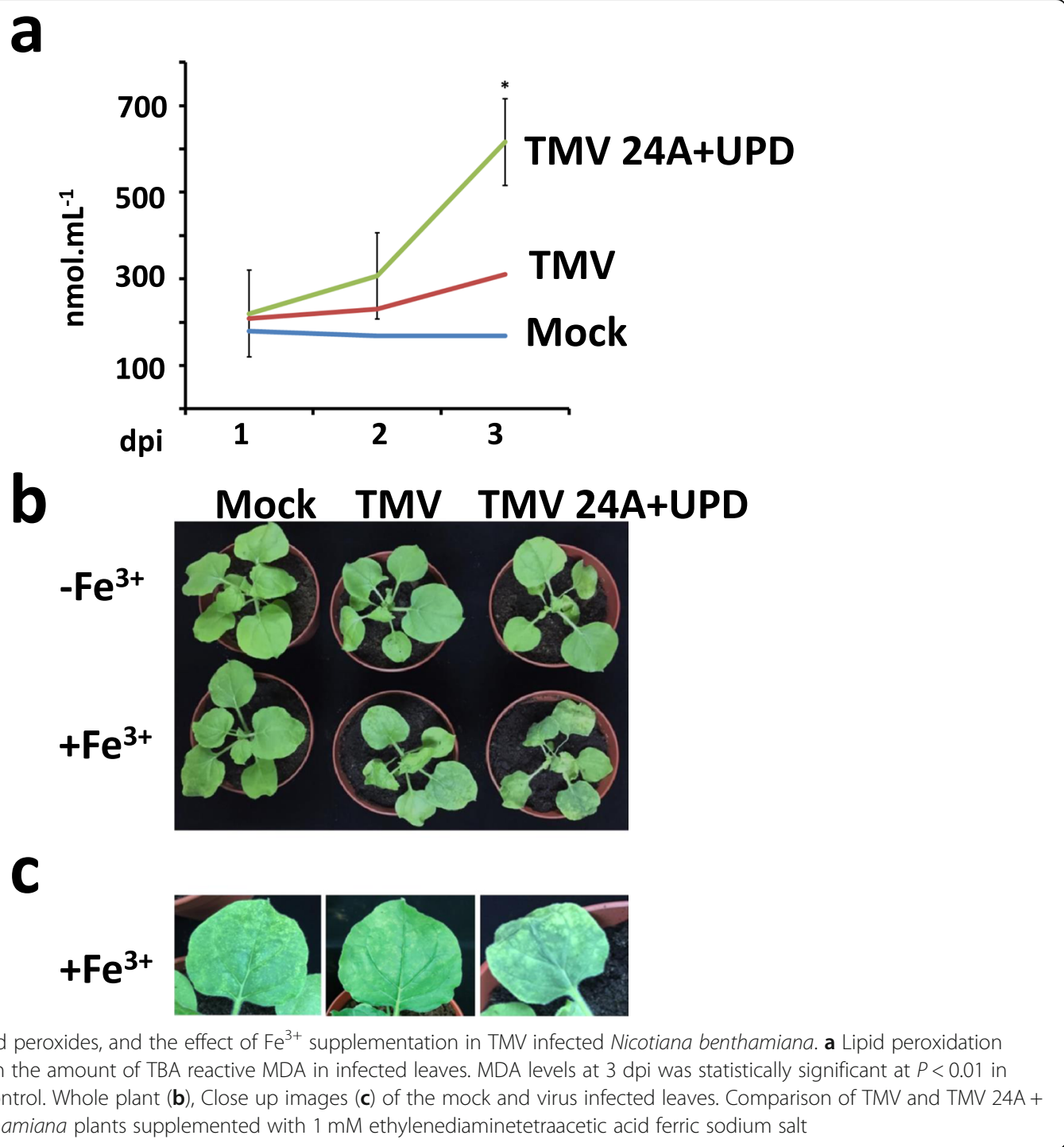

Our results showed that Fer-1 and Lip-1 inhibited the strong cell death response elicited in TMV 24A + UPD inoculated plants (Fig. 7a). We observed that inoculated leaves (shown by red arrows) appeared water soaked and shrivelled in the absence of inhibitors and plants collapsed on the flower pot (Fig. 7a). Mock and TMV inoculated plants and leaves treated with inhibitors did not show such symptom of cell death (Fig. 7a).

We then sought to silence glutathione peroxidase 4 gene (NbGPX4), a gene implicated in ferroptosis (Conrad et al. 2018). In plants, glutathione peroxidase has several isoforms. We have identified phospholipid hydroperoxide (Q9FXS3-GPX4) of Nicotiana tabacum from uniprot. We cloned a gene from $N$. benthamiana which has a $100 \%$ nucleotide sequence identity to $N$. tabacum GPX4 (XM_016605968.1, GQ354813.1). A BLAST search showed that NbGPX4 shared high sequence similarity to HsGPX4 (NM_001367832.1), MmGPX4 (NM_
001367995.1) and AtGPX6 (NM_117229.4), all of which have been ascribed to ferroptosis regulation. Thus, we selected $N b G P X 4$ for this study.

TMV 24A + UPD infected plants exhibited enhanced cell death symptoms in NbGPX4-silenced plants (Fig. 7b). We did not observe such enhanced symptoms in TMV inoculated plants (Fig. 7b). We conclude that a distinct form of ferroptosis-like cell death occurs in TMV 24A + UPD infected plants.

Using various approaches, our study revealed some physiological features of ferroptosis in TMV 24A + UPD infected $N$. benthamiana plants (Fig. 8) as previously described (Dixon et al. 2012). We assessed ROS by quantifying malondialdehyde (MDA) content in infected plants, manipulated intracellular iron by supplementing with iron, employing iron chelators and by virus induced gene silencing (VIGS) of iron storage protein ferritin gene. We also employed potent 


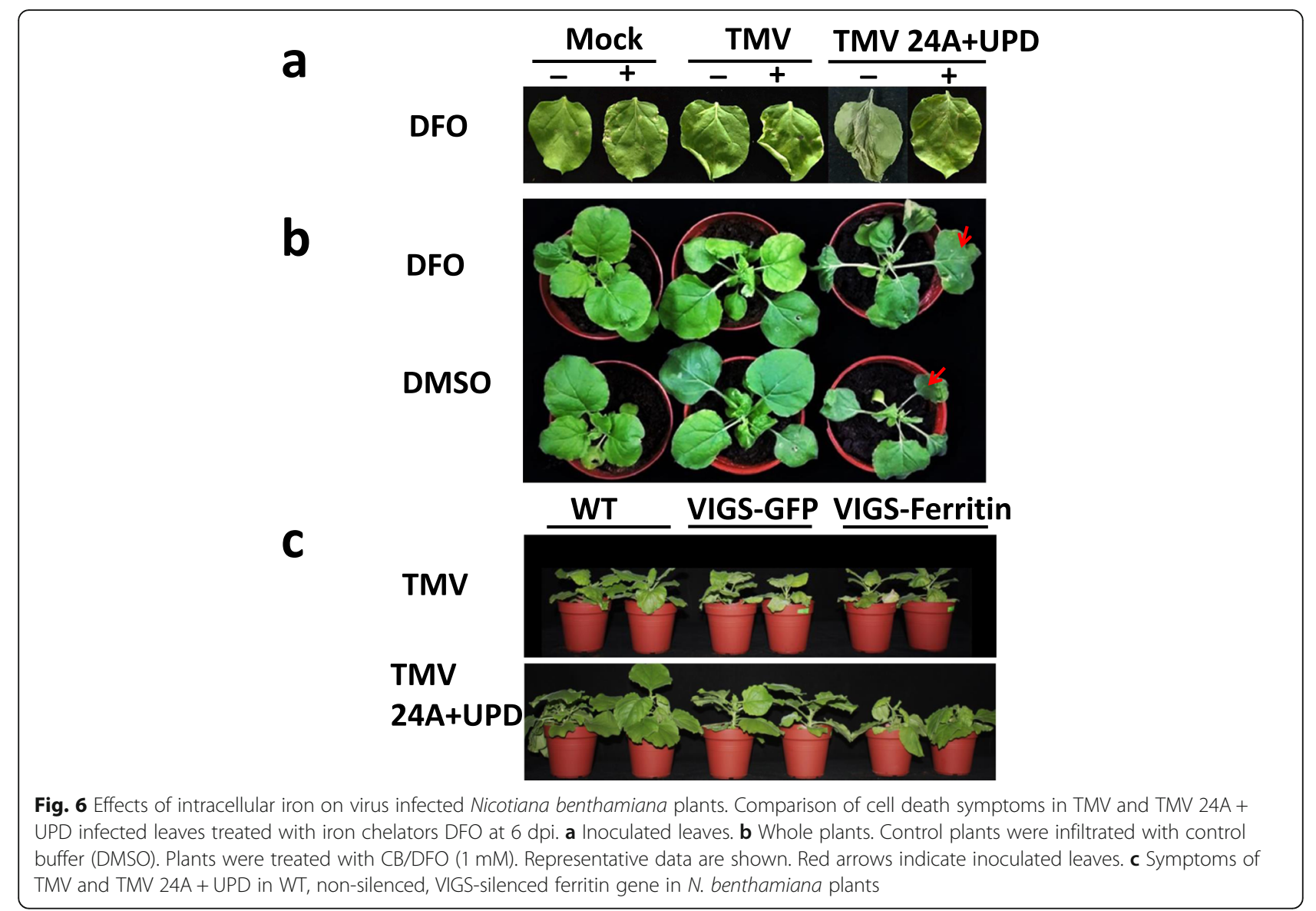

ferroptosis inhibitors ferostatin-1, liprostatin- 1 and silenced GPX4 by VIGS.

\section{Discussion}

Isobaric tag for relative and absolute quantitation LCMS/MS (liquid chromatography tandem mass spectrometry) technology has been used to study virus-host interactions. Previous tudies have compared virus-infected plants with healthy plants to understand these interactions (Wang et al. 2015; Alexander and Cilia 2016; Chen et al. 2017). In this study, we compared the proteome profiles of plants infected with TMV and a fasterreplicating mutant TMV 24A + UPD. The faster replicating mutant induced cell death earlier than TMV. Our aim was to compare proteins involved in cell death from different defense pathways by TMV and its faster replicating mutant TMV 24A + UPD. This study focused on specific proteins that are the cause of accelerated cell death in TMV 24A + UPD treated plants. We therefore compared the proteome profiles of TMV- or TMV 24A + UPD infected N. benthamiana plants at two different time points ( 2 and $3 \mathrm{dpi}$ ) that correlate with the first signs of virus-induced cell death.
The timing and intensity of response to virus infection is crucial to protection against cell death in plants (De Vos et al. 2005). The type and specificity of the plants responses such as regulated cell death also varied accordingly. TMV and TMV 24A + UPD induced defense proteins differed in abundance. The proteome profile of TMV 24A + UPD infected plants revealed a network of defense responses including proteasome, Golgi membrane, mitogen-activated protein kinase (MAPK) and glutathione metabolism (Neumann et al. 2003; Koornneef and Pieterse 2008; País et al. 2009). ROS regulators were identified in protein profiles of both TMV and TMV 24A + UPD infected plants. There were more ROS associated proteins found with TMV 24A + UPD infected plants, as compared to that of TMV (Fig. 4a, b). Peroxiredoxin and glutathione peroxidase were highly abundant only in TMV 24A + UPD infected plants. TMV 24A + UPD appears to induce higher oxidative stress in comparison to TMV. While ROS regulators protect plants from oxidative stress, excessive amount of ROS can cause rapid cell death. Plants have other mechanisms such as RNA silencing to counter invading viruses. Plants infected with TMV showed high abundance of P-body, but TMV 24A + UPD infected plants did 


\section{a}

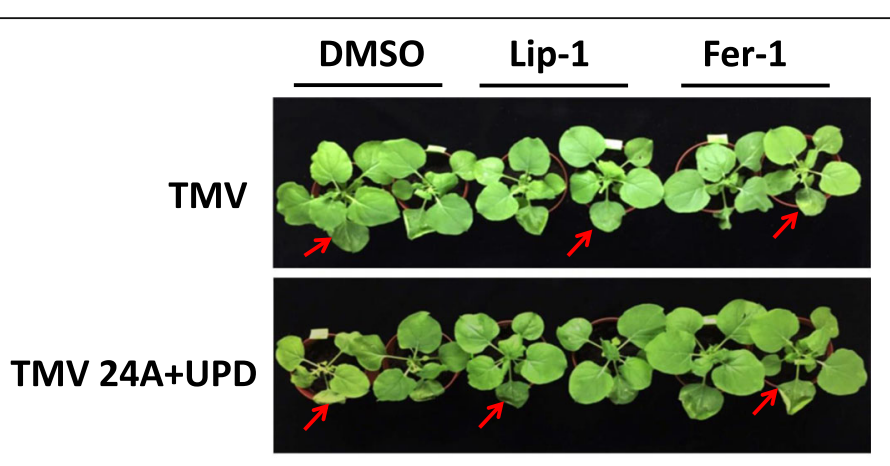

b

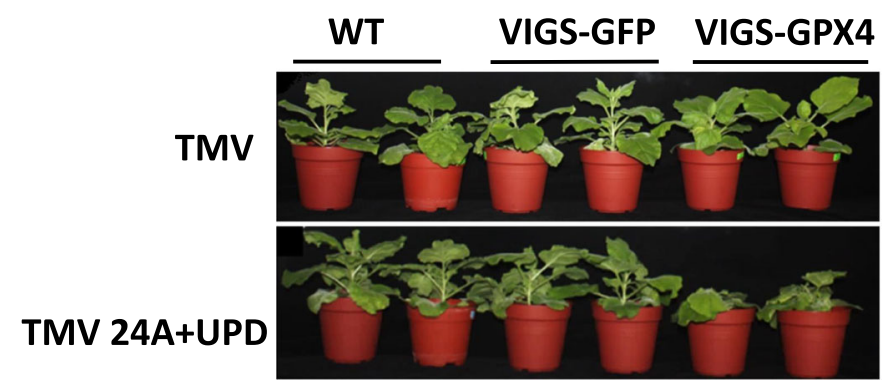

Fig. 7 Effect of ferroptosis inhibition in virus infected Nicotiana benthamiana plants. a Comparison of cell death symptoms in TMV and TMV 24A + UPD plants treated with ferroptosis inhibitors Lip-1 and Fer-1 at 6 dpi. Control plants were infiltrated with DMSO. Plants were treated with DMSO or Fer-1 $(50 \mu \mathrm{M})$ or Lip-1 $(20 \mu \mathrm{M})$. Experiments were performed two times. Representative data are shown. Red arrows show inoculated leaves. $\mathbf{b}$ The symptoms of TMV and TMV 24A + UPD in WT, non-silenced, NbGPX4-silenced N. benthamiana. Transcription of gene(s)-of-interest was downregulated via TRV-induced gene silencing

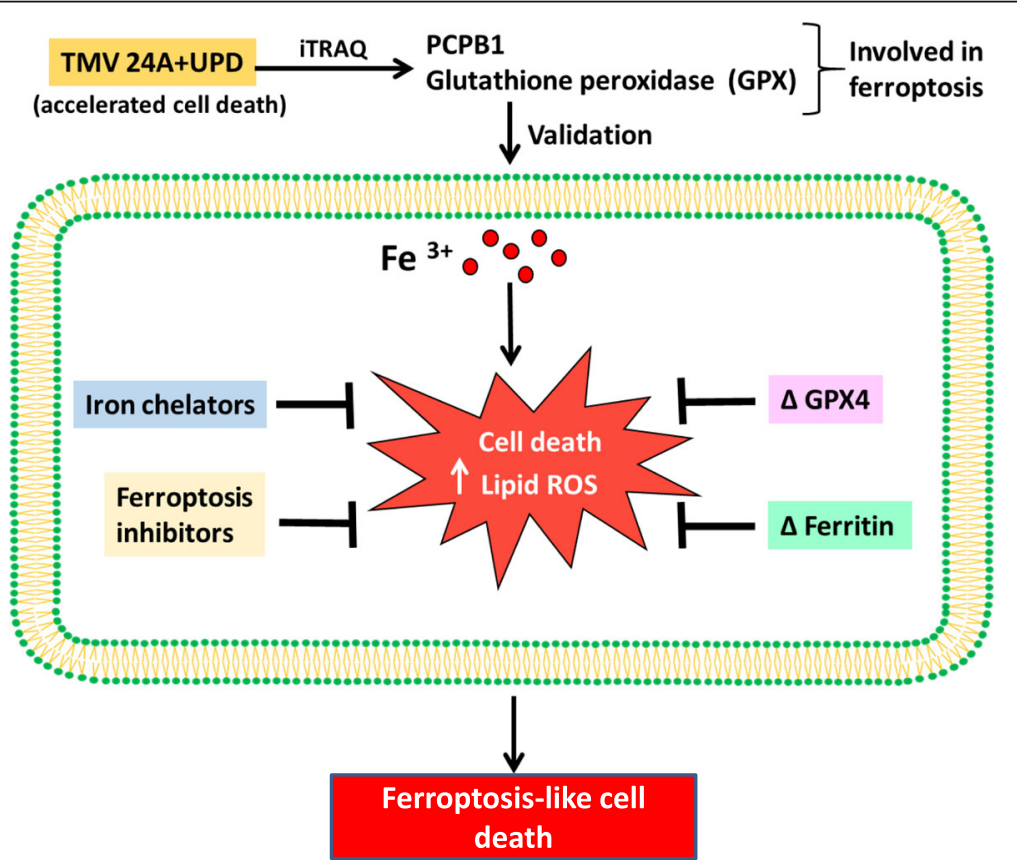

Fig. 8 Ferroptosis-like cell death in TMV 24A + UPD infected Nicotiana benthamiana plants. Using iTRAQ, unique DAP's in the TMV 24A + UPD profile under the ferroptosis and glutathione metabolism pathways were identified. TMV 24A + UPD infected plants showed accelerated cell death symptoms when intracellular iron was increased. Decreasing intracellular iron by employing iron chelators DFO, CPX and silencing of iron storage protein ferritin gene protected the plants from accelerated cell death. We also observed a decrease in TMV 24A + UPD induced cell death when we applied ferroptosis inhibitors ferostatin-1, liprostatin-1. Glutathione peroxidase 4 gene-silenced plants showed enhanced cell death compared to non-silenced control plants 
not. P-body plays a crucial role in RNA silencing (Eulalio et al. 2007). RNA silencing as a conserved defense pathway, evolves within plants against invading viruses. RNA interference (RNAi) suppression increases virus replication and cell death in mosquitoes (Cirimotich et al. 2009). It suppresses virus replication by targeting the secondary structure of positive-sense RNA genome (Szittya et al. 2002; Molnar et al. 2005). TMV 24A + UPD is an artificial mutant that has an altered secondary structure compared to that of TMV (Guo et al. 2015). It is plausible that TMV 24A + UPD RNA secondary structure suppresses RNAi efficiency and leads to enhanced virus replication and host cell death. Our finding suggests that TMV 24A + UPD enhanced plant defense response which led to more rapid cell death in infected plants.

Differentially abundant proteins identified were grouped into three major cell death pathways; apoptosis, necroptosis and ferroptosis (Additional file 2: Table S1). In plants, apoptosis is not markedly distinctive as compared to that of animal cells (Vanyushin et al. 2004). In this study, we did not find any significant numbers of apoptosis-related proteins. The differentially abundant proteins between TMV and TMV 24A + UPD-infected plants were comparable. Cytochrome $\mathrm{C}$ was linked to apoptosis in the protein profile of TMV-infected plants, while peroxiredoxin was linked to that of TMV 24A + UPD-infected plants (Additional file 2: Table S1). Peroxiredoxin guards against oxidative stress caused by abundance of peroxides (Perkins et al. 2015). Cytochrome C released from mitochondria is associated with initiation of programme cell death in plants (Martínez-Fábregas et al. 2013). It is possible that both TMV and TMV $24 \mathrm{~A}+\mathrm{UPD}$ can induce apoptosis in cells of infected plants. Necroptosis associated proteins were high in abundance in TMV 24A + UPD infected plants, but not with TMV. Necroptosis is associated with elevated levels of ROS (Zhou et al. 2012). It results in cell death that leads to uncontrolled disorganization of cellular contents through cell membrane rupture (Dickman and de Figueiredo 2013). Necroptosis is mediated by a complex of proteins that make up the necrosome. It is possible to switch from apopotosis to necroptosis using factors such as autophagy in mammalian cells (Goodall et al. 2016). A similar switch likely exists in plants. Ferroptosis associated proteins were highly abundant in TMV $24 \mathrm{~A}+$ UPD infected plants. Ferroptosis is first described in mammalian cells (Dixon et al. 2012). It is morphologically and genetically distinct from other forms of cell death (Hao et al. 2018). In plants, ferroptosis-like cell death has been reported in Arabidopsis root hairs subjected to $55^{\circ} \mathrm{C}$ heat shock (Distéfano et al. 2017). It is also involved in R-gene mediated resistance against rice blast fungus Magnaporthe oryzae (Dangol et al. 2018). This indicates that ferroptosis can be a conserved pathway for both plants and animals. These results also indicate that cell death from TMV infection can arise from different defense pathways in $N$. benthamiana plants.

One of the characteristics of ferroptosis is the increased accumulation of intracellular iron. TMV 24A + UPD induced higher abundance of oxidative stress proteins such as the ROS regulators in infected $N$. benthamiana plants. Many anti-oxidative enzymes require iron to catalyse electron transport (Tewari et al. 2005). Our data revealed that cell death in TMV 24A + UPD infected plants was inhibited in leaves infiltrated with iron chelators. Iron chelators have been shown to protect Arabidopsis against Pseudomonas syringae pv. tomato DC3000 (Aznar et al. 2014). An increase in intracellular iron by exogenous supplementation led to further accelerated cell death in TMV 24A + UPD infected plants. Ferritin protein regulates intracellular iron to protect plants against oxidative stress (Ravet et al. 2009). Reduced transcription levels of ferritin by VIGS in $N$. benthamiana plants further accelerated cell death in TMV 24A + UPD infected plants. We infer that intracellular iron is functionally associated with ferroptosis-like cell death in $N$. benthamiana plants infected with the fast-replicating mutant TMV 24A + UPD.

Another characteristic of ferroptosis is iron induced lipid peroxidation (Conrad et al. 2018). Lipid peroxidation in biological membranes is an indicator of oxidative stress in plants. Iron deficient plants have been shown to have decreased lipid peroxidation (Tewari et al. 2005). We quantified the MDA content using TBARS assay. While MDA has been used to ascertain ferroptosis associated lipid peroxidation in plants (Dangol et al. 2018), it is not exclusively generated by lipid peroxidation. We thus further employed ferroptosis inhibitors to ascertain lipid peroxidation and its role in cell death. Ferroptosis has been characterized using inhibitors ferrostatin-1 and liproxstatin-1 (Friedmann Angeli et al. 2014; Linkermann et al. 2014; Distéfano et al. 2017; Dangol et al. 2018). We observed decreased cell death in Fer-1/Lip-1 treated plants which were infected with TMV 24A + UPD. We note that in this study, we used higher concentration of ferroptosis inhibitors relative to other studies (Distéfano et al. 2017; Dangol et al. 2018). Our study utilizes infiltration of the inhibitors in mature leaves while other studies use inhibitors in growth media. We determined optimal concentration to determine the effect of the inhibitors on cell death and avoided concentration that triggered a necrotic cell death response.

To further ascertain lipid peroxidation, we knocked down GPX4 by VIGS. GPX4 is the major antioxidant associated with ferroptosis. GPX4 knockdown in mice has been shown to increase lipid peroxidation dependent cell death (Imai et al. 2017). We observed accelerated cell death in GPX4 knockdown plants infected with TMV 24A + UPD. 
TMV induces cell death in $N$. benthamiana with symptoms of rapid leaf necrosis and stem collapse, leading to plant death within a few days. We assessed these symptoms to show decrease or acceleration of cell death symptoms when we applied inhibitors, increased iron concentration and silenced GPX4 and ferritin gene. As these symptoms are not exclusively associated with ferroptosis, we do not rule out other forms of cell death in addition to the ferroptotic-like cell death inferred from this study. Taken together, our data suggest that TMV 24A + UPD can induce ferroptosis-like programmed cell death in $N$. benthamiana plants.

\section{Conclusions}

We employed the iTRAQ-based quantitative proteomics approach to determine the proteomes of TMV and TMV 24A + UPD-infected plants to compare proteins involved in cell death from different defense pathways by TMV and its faster replicating mutant TMV 24A + UPD. This study provides evidence that TMV 24A + UPD accelerated cell death involves several pathways in $N$. benthamiana plants. KEGG analysis revealed differences in differentially abundant proteins of the two proteome profiles under cell death, stress signalling, protein folding, sorting, degradation, transport and catabolism. We identified unique differentially abundant proteins in the TMV 24A + UPD profile, in particular under the ferroptosis and glutathione metabolism pathways. We showed a link between intracellular iron and accelerated lipid ROS induced cell death in TMV 24A+ UPD infected plants. These results suggest that this TMV mutant induces a distinct form of cell death similar to that of ferroptosis.

\section{Methods}

\section{Plant material and virus inoculation}

A total of 32 four-week-old $N$. benthamiana plants were used for this study. TMV U1 was used for this study. All plants were grown under $16 \mathrm{~h}$ light and $8 \mathrm{~h}$ dark at $25{ }^{\circ} \mathrm{C}$. Fully expanded leaves were dusted with carborundum and mechanically inoculated with $2.5 \mu \mathrm{g}$ of in vitro transcribed RNA in GKP buffer (50 mM glycine; $30 \mathrm{mM}$ $\mathrm{K}_{2} \mathrm{HPO}_{4}, \mathrm{pH}$ 9.2; $1 \%$ bentonite; $1 \%$ celite).

\section{Leaf protein preparation}

Total protein was extracted from the inoculated and newly emerged leaves of TMV and TMV 24A + UPD treated plants at 2 and $3 \mathrm{dpi}$, respectively. For each time point, four biological replicates were included in the samples and grouped into two iTRAQ tagging (Fig. 1). Total protein was extracted and quantified using the Bradford assay (Das et al. 2018). Western blotting was used to confirm virus infection using antisera against TMV coat protein.

\section{Sample preparation}

The protein concentration was determined using Quick Start $^{\text {tm }}$ Bradford Protein Assay and normalized to $100 \mu \mathrm{g}$ for all the samples. The protein samples were directly incorporated into a polyacrylamide gel matrix without electrophoresis as described (Lu and Zhu 2005). Briefly, proteins were immobilized in a gel matrix and then reduced with Tris (2carboxyethyl) phosphine (TCEP) and then alkylated with methyl methanethiosulfonate (MMTS). Trypsin in-gel digestion was carried out at $37^{\circ} \mathrm{C}$ for $16 \mathrm{~h}$. The proteins were extracted from the gel using $0.5 \mathrm{M}$ triethylammonium bicarbonate (TEAB), $\mathrm{pH}$ 8.5. The samples were processed for iTRAQ by labelling with iTRAQ 8PLEX kit (ABI-SCIEX), following the manufacturer's protocol.

\section{iTRAQ labelling and tandem mass spectrometry}

A total of eight samples were tagged (Fig. 1). Labelling was performed as described (Meng et al. 2014). The samples were pooled together for an 8-plex experiment. Sample desalting was carried out using C18 Sep-Pak (Pierce) cartridge followed by protein lyophilisation. Lyophilized protein samples were prepared by dissolving in $1 \mathrm{~mL}, 20 \mathrm{mM}$ ammonium formate in water, $\mathrm{pH} 10$. For downstream LC-ESI-MS/MS analysis, iTRAQ labelled peptides were subjected to reverse-phase high performance liquid chromatography (RP-HPLC) equipped with C18 column. Mobile phase A comprises of $20 \mathrm{mM}$ ammonium formate water, while the mobile phase $\mathrm{B}$ consists of $80 \%$ acetonitrile (ACN). Fractions were collected at 1 -min intervals with the flow rate set at $0.5 \mathrm{~mL} / \mathrm{min}$. The eluents were pooled into five fractions, lyophilized and reconstituted in $2 \% \mathrm{ACN}$ in water. Each fraction was subjected to MS analysis on a 5600 Triple TOF system (SCIEX). The precursor ion selection parameters were set at mass range $400-1800 \mathrm{~m} / \mathrm{z}$ and accumulation time $250 \mathrm{~ms} / \mathrm{sec}$.

Data analysis was performed as described by Meng et al. (2014). MS/MS data were searched against protein sequence database Nicotiana using ProteinPilot ${ }^{\text {tw }}$ software. The protein ratios were weighted and normalized by the median ratio. The ratios with $p$ values $<0.05$, and/or fold changes $>1.5$ (up-regulation/highly abundant) and $<0.67$ fold (down-regulation/low abundance) were considered significant. Identification and classification of differentially regulated proteins of TMV and TMV 24A + UPD were analysed separately by comparing the protein profiles at 3 dpi against 2 dpi.

\section{Bio-informatics analysis}

Differentially abundant proteins (DAPs) were functionally classified by first assigning GO and $\mathrm{KO}$ ID using eggnog-mapper (http://eggnogdb.embl.de/\#/app/emapper) against eggnog 4.5.1 database, within the taxonomic 
group Streptophyta. The Gene Ontology interactive network were summarized and plotted following published REVIGO protocol (http://revigo.irb.hr. KEGG pathway analysis was conducted using KEG mapperhttp://www. genome.jp/kegg/tool/map_pathway.html). Venn diagrams were generated using UGen Venn software (http://bioinformatics.psb.ugent.be/webtools/Venn/).

\section{Validation of differentially abundant proteins using real time PCR analysis}

Total RNA was isolated from pooling 4 replicate samples for each treatment. The quality and quantity were confirmed by agarose gel electrophoresis and NanoDrop ${ }^{\mathrm{Tn}}$ measurement, respectively. RNA was converted into cDNA by reverse transcription using oligo $d(T)$ primer. All Primers used are shown (Additional file 2: Table S2). Quantitative real-time RT-PCR was performed to determine the expression of selected host genes of interest, using the KAPA SYBR FAST universal qPCR kit and CFX384TM real time PCR detection system (Bio-Rad).

Housekeeping genes 5.8S rRNA gene and actin were used as internal controls in the calculation of relative transcript levels at 2 and 3 dpi with TMV and TMV 24A + UPD. Relative quantification of transcripts was conducted using comparative CT method ( $2^{-\Delta \Delta C T}$ method). The experiment was conducted for all biological replicates with three technical triplicates for each sample.

\section{Molecular cloning and quantitative PCR}

Ferritin and GPX4 genes were amplified from $N$. benthamiana cDNA using primers NtFerrF/R and NbGPX4F/R (Additional file 2: Table S3). Cloning of fragments into vector PTRV2 (VIGS) was conducted using Gibson Assembly (GA) cloning kit from NEB. Primers were designed to overlap both the vector and cloned PCR fragments. Quantitative real-time (qRT-PCR) to determine GPX4 and ferritin gene expression levels was performed. The primers used were DR_qGPX4 F/R, DR qFerF/R and qGPX4F/R (Additional file 2: Table S3).

\section{Virus-induced gene silencing}

Amplified target gene fragments were inserted into pTRV2. Together, pTRV1 and pTRV2 were introduced into Agrobacterium by electroporation. Agrobacteria containing pTRV1 and pTRV2 or pTRV2 derivative plasmids were grown overnight at $28^{\circ} \mathrm{C}$. Bacteria were resuspended in agroinfiltration buffer and mixed at 1:1 ratio as previously described (Liu et al. 2002). After 4-h incubation at room temperature, the mixed agrobacteria cultures were infiltrated into the leaves of 6-leaf-stage $N$. benthamiana plants. Silenced phenotypes appeared in the upper leaves approximately 10 days post-infiltration. Gene silencing was confirmed by qRT-PCR in the silenced plants. It showed significant down-regulation of the targeted genes relative to non-silenced plants (Additional file 5: Figure S4).

\section{Analyzing the role of ferroptosis in TMV-infected plants} Deferoxamine (deferoxamine mesylate salt; DFO) and ciclopirox olamine (CPX) act as iron chelators, while ferrostatin-1 (Fer-1) and liproxstatin-1 (Lip-1) are potent inhibitors of ferroptosis. The chelators/inhibitors were used at the following concentrations: DFO $(1 \mathrm{mM}), \mathrm{CPX}$ $(250 \mu \mathrm{M})$, Fer-1 $(50 \mu \mathrm{M})$ and Lip-1 $(20 \mu \mathrm{M})$. First, the plants were inoculated with viral RNA as previously described and then infiltrated with the reagents above after $12 \mathrm{~h}$.

The solution was infiltrated into fully expanded leaves at six-leaf stage using a syringe without a needle. Control plants were infiltrated with DMSO. On each plant, three leaves were infiltrated. Four plants were used for each experiment. Experiments were performed three times.

\section{Thiobarbituric acid reactive substances (TBARS) assay}

Estimation of lipid peroxidation in infected $N$. benthamiana leaves was assayed using the measurement of malondialdehyde (MDA) content (Heath and Packer 1968). Briefly, $3 \mathrm{~mL}$ of trichloroacetic acid (TCA) was added into $500 \mathrm{mg}$ of leaf tissue and ground up with a pestle and mortar. The resulting homogenate was centrifuged at $10,000 \times \mathrm{g}$ for $10 \mathrm{~min}$. The supernatant was transferred to a fresh tube. One $\mathrm{mL}$ of the supernatant was added into $4 \mathrm{~mL}$ of a mixture of $20 \%$ TCA and $0.5 \%$ thiobarbituric acid (TBA). The mixture was capped tightly and heated in a water bath at $95^{\circ} \mathrm{C}$ for $30 \mathrm{~min}$ and then quickly cooled on ice. The cooled mixture was centrifuged at $10,000 \times \mathrm{g}$ for $15 \mathrm{~min}$.

The supernatant was transferred into cuvettes and the absorbance was determined using a spectrophotometer at $532 \mathrm{~nm}$. The concentration of MDA was calculated using the Beer Lamberts formula $\mathrm{A}=\Sigma \mathrm{lc}$ : $\mathrm{A}=\mathrm{Absorb}-$ ance, $\mathcal{E}=$ extinction coefficient, $1=$ path length. The extinction coefficient of MDA is known to be $155 \mathrm{mM}^{-1} \mathrm{~cm}^{-1}$ (Hodges et al. 1999).

\section{$\mathrm{Fe}^{3+}$ supplementation}

We supplemented iron to experimental plants through soil by irrigating $N$. benthamiana plants with ethylenediaminetetraacetic acid ferric sodium solution at a final concentration of $1 \mathrm{mM}$. Iron is assimilated by the roots from the soil (Briat et al. 2007; Kim and Guerinot 2007). After two rounds of irrigation with equal amount of water, the plants were inoculated with virus. Mock plants did not include ethylenediaminetetraacetic acid ferric sodium salt in the irrigation water. Experiments were performed three times. 


\section{Supplementary information}

Supplementary information accompanies this paper at https://doi.org/10. 1186/s42483-019-0043-5

Additional file 1: Figure S1. Validation of iTRAQ through qRT-qPCR. Fold changes in the mRNA. TMV (a), TMV 24A + UPD (b) infected plants. SGT1 (small glutamine rich tetratricopeptide repeat containing beta), GSTP1 (glutathione transferase), 26SPS (26S proteasome), HIR1 (Hypersensitive-induced response protein 1-like), EF1 (Elongation factor 1-alpha), GAPDH (Glyceraldehyde 3-phosphate dehydrogenase), gyrB (DNA gyrase subunit B), CSP41B (Chloroplast stem-loop binding protein of $41 \mathrm{kDa}$ ), CB2A (Chlorophyll a-b binding protein), 28 kRNP (28 kDa ribonucleoprotein, plastid), PEX 14 (Peroxisomal membrane protein PEX14-like), PRXIIE-2 (Peroxiredoxin-2e-2), PDCD4 (Programmed cell death protein 4), ERG3-L (Elicitor-responsive protein 3-like), PEVMPP (Pyrophosphate-energized vacuolar membrane proton pump), V-ATPase (V-type proton ATPase), API5(Apoptosis inhibitor 5-like), NST1(Stress response protein), psaA (Photosystem I P700), EMC4 (ER membrane protein 4), DRT111(DNA-damage-repair protein).

Additional file 2: Table S1. Selected proteins identified from KEGG pathways. Table S2. Primers used in qRT-PCR validation. Table S3. Primers used in cloning and PCR.

Additional file 3: Figure S2. Comparison of cell death symptoms in TMV and TMV 24A + UPD infected plants treated with CPX at 6 dpi.

Additional file 4: Figure S3. Effect of GPX4 and ferritin gene silencing in N. benthamiana plants infected with TMV 24A + UPD at $12 \mathrm{dpi}$.

Additional file 5: Figure S4. Analysis of gene downregulation in VIGS plants.

\section{Abbreviations}

26SPS: $26 S$ proteasome; 28 kRNP: 28 kDa ribonucleoprotein, plastid: ACN: Acetonitrile; API5: Apoptosis inhibitor 5-like; BCIP: 5-bromo-4-chloro-3indolyl phosphate; CB2A: Chlorophyll a-b binding protein;

CSP41B: Chloroplast stem-loop binding protein of 41 kDa; DAPs: Differentially abundant proteins; DRT111: DNA-damage-repair protein; EF1: Elongation factor 1-alpha; EMC4: ER membrane protein 4; ERG3-L: Elicitor-responsive protein 3-like; FDR: False discovery rate; GAPDH: Glyceraldehyde 3-phosphate dehydrogenase; GO: Gene ontology; GSTP1: Glutathione transferase; gyrB: DNA gyrase subunit B; HIR1: Hypersensitive-induced response protein 1-like; iTRAQ: Isobaric tags for relative and absolute quantification; KEGG: Kyoto Encyclopedia of Genes and Genomes; LC-MS/MS: Liquid chromatography-tandem mass spectrometry; MDA: Malondialdehyde; MMTS: Methyl methanethiosulfonate; NBT: Nitroblue tetrazolium; NST1: Stress response protein; PBS: Phosphate buffered saline; PCBP1: Poly (rC)-binding protein 1; PCR: Quantitative real time polymerase chain reaction; PDCD4: Programmed cell death protein 4; PEVMPP: Pyrophosphateenergized vacuolar membrane proton pump; PEX 14: Peroxisomal membrane protein PEX14-like; PMSF: Phenylmethylsulfonyl fluoride; psaA: Photosystem I P700; PRXIIE-2: Peroxiredoxin-2e-2; ROS: Reactive oxygen species; RP-HPLC: Reverse-phase high-performance liquid chromatography; SDS: Sodium dodecyl sulphate; SGT1: Small glutamine-rich tetratricopeptide; TBA: Thiobarbituric acid; TBARS: Thiobarbituric acid reactive substances; TCEP: Tris(2-carboxyethyl)phosphine; TEAB: Triethylammonium bicarbonate; TMV: Tobacco mosaic virus; UPD: Upstream pseudoknot domain; UTR: Untranslated region; V-ATPase: V-type proton ATPase

\section{Acknowledgements}

The authors would like to thank Mr. Lim Teck Kwang at Protein and Proteomics Center, Department of Biological Sciences, NUS for his technical support and Dr. Timothy Holton for comments on the first draft of the manuscript.

\section{Authors' contributions}

MM performed experiments, analysis and writing of the manuscript. PPD, NIN and S-MW assisted in design of the study, data analysis and interpretation of results, and in editing the manuscript. All authors read and approved the final manuscript.

\section{Funding}

This work was financially supported by Singapore Ministry of Education through National University of Singapore research grant R-154-000-B23-114, Suzhou Bureau of Science and Technology grant SNG201839, National Natural Science Foundation of China grant 31872639 and NUS-SINGA scholarship to Mercy Macharia. Naweed Naqvi acknowledges intramural funding from the Temasek Life Sciences Laboratory, Singapore.

Availability of data and materials

Not applicable.

Ethics approval and consent to participate

Not applicable.

\section{Consent for publication}

Not applicable.

\section{Competing interests}

The authors declare that they have no competing interests.

\section{Author details}

${ }^{1}$ Department of Biological Sciences, National University of Singapore, Singapore 119543, Singapore. ${ }^{2}$ Temasek Life Sciences Laboratory, Singapore 117604, Singapore. ${ }^{3}$ National University of Singapore Research Institute, Suzhou, Jiangsu 215123, People's Republic of China.

Received: 26 October 2019 Accepted: 18 December 2019 Published online: 06 January 2020

\section{References}

Alexander MM, Cilia M. A molecular tug-of-war: global plant proteome changes during viral infection. Curr Plant Biol. 2016:5:13-24.

Aznar A, Chen NWG, Rigault M, Riache N, Joseph D, Desmaële D, et al. Scavenging iron: a novel mechanism of plant immunity activation by microbial siderophores. Plant Physiol. 2014;164:2167-83.

Briat JF, Curie C, Gaymard F. Iron utilization and metabolism in plants. Curr Opin Plant Biol. 2007:10:276-82

Chen H, Cao Y, Li Y, Xia Z, Xie J, Carr JP, et al. Identification of differentially regulated maize proteins conditioning Sugarcane mosaic virus systemic infection. New Phytol. 2017;215:1156-72.

Cirimotich CM, Scott JC, Phillips AT, Geiss BJ, Olson KE. Suppression of RNA interference increases alphavirus replication and virus-associated mortality in Aedes aegypti mosquitoes. BMC Microbiol. 2009;9:49.

Conrad M, Kagan VE, Bayir H, Pagnussat GC, Head B, Traber MG, et al. Regulation of lipid peroxidation and ferroptosis in diverse species. Genes Dev. 2018;32:602-19.

Dangol S, Chen Y, Hwang BK, Jwa NS. Iron- and reactive oxygen species-dependent ferroptotic cell death in rice-Magnaporthe oryzae interactions. Plant Cell. 2018;1:189-09.

Das PP, Lin Q, Wong S-M. Comparative proteomics of Tobacco mosaic virusinfected Nicotiana tabacum plants identified major host proteins involved in photosystems and plant defence. J Proteome. 2018:94:191-9.

De Vos M, Van Oosten VR, Van Poecke RM, Van Pelt JA, Pozo MJ, Mueller MJ, et al. Signal signature and transcriptome changes of Arabidopsis during pathogen and insect attack. Mol Plant-Microbe Interact. 2005;18:923-37.

Di Carli M, Benvenuto E, Donini M. Recent insights into plant-virus interactions through proteomic analysis. J Proteome Res. 2012;11:4765-80.

Dickman MB, de Figueiredo P. Death be not proud_cell death control in plant fungal interactions. PLoS Pathog. 2013;9:e1003542.

Distéfano AM, Martin MV, Córdoba JP, Bellido AM, D'lppólito S, Colman SL, et al. Heat stress induces ferroptosis-like cell death in plants. J Cell Biol. 2017;16:463-76.

Dixon SJ, Lemberg KM, Lamprecht MR, Skouta R, Zaitsev EM, Gleason CE, et al. Ferroptosis: an iron-dependent form of nonapoptotic cell death. Cell. 2012; 149:1060-72.

Elmore S. Apoptosis: a review of programmed cell death. Toxicol Pathol. 2007;35:495-516 Eulalio A, Behm-Ansmant I, Schweizer D, Izaurralde E. P-body formation is a consequence, not the cause, of RNA-mediated gene silencing. Mol Cell Biol. 2007;27:3970-81.

Friedmann Angeli JP, Schneider M, Proneth B, Tyurina YY, Tyurin VA, Hammond $\mathrm{VJ}$, et al. Inactivation of the ferroptosis regulator Gpx4 triggers acute renal failure in mice. Nat Cell Biol. 2014;16:1180-91.

Garcia-Ruiz H. Susceptibility genes to plant viruses. Viruses. 2018;10:484.

Goodall ML, Cramer SD, Thorburn A. Autophagy complexes cell death by necroptosis. Oncotarget. 2016;7:50818-9. 
Guo S, Kierzek E, Chen G, Zhou YJ, Wong SM. TMV mutants with poly(a) tracts of different lengths demonstrate structural variations in 3'UTR affecting viral RNAs accumulation and symptom expression. Sci Rep. 2015;5:18412.

Hao S, Liang B, Huang Q, Dong S, Wu Z, He W, et al. Metabolic networks in ferroptosis. Oncol Lett. 2018;15:5405-11.

Heath RL, Packer L. Photoperoxidation in isolated chloroplasts: I. kinetics and stoichiometry of fatty acid peroxidation. Arch Biochem Biophys. 1968;125:189-98.

Hodges DM, DeLong JM, Forney CF, Prange RK. Improving the thiobarbituric acidreactive-substances assay for estimating lipid peroxidation in plant tissues containing anthocyanin and other interfering compounds. Planta. 1999;207:604-11.

Imai H, Matsuoka M, Kumagai T, Sakamoto T, Koumura T. Lipid peroxidationdependent cell death regulated by GPx4 and ferroptosis. In: Nagata S, Nakano H, editors. Apoptotic and non-apoptotic cell death. Current topics in microbiology immunology, vol. 403. Cham: Springer Nature; 2017. p. 143-70.

Kim SA, Guerinot ML. Mining iron: iron uptake and transport in plants. FEBS Lett. 2007:581:2273-80.

Koornneef A, Pieterse CM. Cross talk in defense signaling. Plant Physiol. 2008;146:839-44.

Leidgens S, Bullough KZ, Shi H, Li F, Shakoury-Elizeh M, Yabe T, et al. Each member of the poly-r(C)-binding protein 1 (PCBP) family exhibits iron chaperone activity toward ferritin. J Biol Chem. 2013;288:17791-802.

Lietzen N, Ohman T, Rintahaka J, Julkunen I, Aittokallio T, Matikainen S, et al. Quantitative subcellular proteome and secretome profiling of influenza a virus-infected human primary macrophages. PLoS Pathog. 2011;7:e1001340.

Linkermann A, Skouta R, Himmerkus N, Mulay SR, Dewitz C, De Zen F, et al. Synchronized renal tubular cell death involves ferroptosis. Proc Natl Acad Sci U S A. 2014;111:16836-41.

Liu Y, Schiff M, Dinesh-Kumar SP. Virus-induced gene silencing in tomato. Plant J. 2002:31:777-86.

Lu X, Zhu H. Tube-gel digestion: a novel proteomic approach for high throughput analysis of membrane proteins. Mol Cell Proteomics. 2005;4:1948-58.

Maiorino M, Conrad M, Ursini F. GPx4, lipid peroxidation, and cell death: discoveries, rediscoveries, and open issues. Antioxid Redox Signal. 2018;29:61-74.

Martínez-Fábregas J, Díaz-Moreno I, González-Arzola K, Janocha S, Navarro JA, Hervás M, et al. New Arabidopsis thaliana cytochrome c partners: a look into the elusive role of cytochrome $\mathrm{c}$ in programmed cell death in plants. Mol Cell Proteomics. 2013;12:3666-76.

Meng Q, Hou L, Zhao Y, Huang X, Huang Y, Xia S, et al. iTRAQ-based proteomic study of the effects of Spiroplasma eriocheiris on Chinese mitten crab Eriocheir sinensis hemocytes. Fish Shellfish Immunol. 2014;40:182-9.

Molnar A, Csorba T, Lakatos L, Varallyay E, Lacomme C, Burgyan J. Plant virusderived small interfering RNAs originate predominantly from highly structured single-stranded viral RNAs. J Virol. 2005;79:7812-8.

Mukhtar MS, McCormack ME, Argueso CT, Pajerowska-Mukhtar KM. Pathogen tactics to manipulate plant cell death. Curr Biol. 2016;26:R608-R19.

Neumann U, Brandizzi F, Hawes C. Protein transport in plant cells: in and out of the Golgi. Ann Bot. 2003;92:167-80.

Niu S, Cao S, Huang $\sqcup$, Tan KC, Wong SM. The length of an internal poly(a) tract of hibiscus latent Singapore virus is crucial for its replication. Virology. 2015;474:52-64.

País SM, Téllez-Iñón MT, Capiati DA. Serine/threonine protein phosphatases type 2A and their roles in stress signaling. Plant Signal Behav. 2009;4:1013-5.

Perkins A, Nelson KJ, Parsonage D, Poole LB, Karplus PA. Peroxiredoxins: guardians against oxidative stress and modulators of peroxide signaling. Trends Biochem Sci. 2015;40:435-45.

Ravet K, Touraine B, Kim SA, Cellier F, Thomine S, Guerinot ML, et al. Posttranslational regulation of AtFER2 ferritin in response to intracellular iron trafficking during fruit development in Arabidopsis. Mol Plant. 2009;2:1095-106.

Reape TJ, Molony EM, McCabe PF. Programmed cell death in plants: distinguishing between different modes. J Exp Bot. 2008;59:435-44.

Seibt TM, Proneth B, Conrad M. Role of GPX4 in ferroptosis and its pharmacological implication. Free Radic Biol Med. 2019:133:144-52.

Skouta R, Dixon SJ, Wang J, Dunn DE, Orman M, Shimada K, et al. Ferrostatins inhibit oxidative lipid damage and cell death in diverse disease models. J Am Chem Soc. 2014;136:4551-6.

Szittya G, Molnar A, Silhavy D, Hornyik C, Burgyan J. Short defective interfering RNAs of tombusviruses are not targeted but trigger post-transcriptional gene silencing against their helper virus. Plant Cell. 2002;14:359-72.

Tewari RK, Kumar P, Neetu, Sharma PN. Signs of oxidative stress in the chlorotic leaves of iron starved plants. Plant Sci. 2005;169:1037-45.

Vanyushin BF, Bakeeva LE, Zamyatnina VA, Aleksandrushkina NI. Apoptosis in plants: specific features of plant apoptotic cells and effect of various factors and agents. Int Rev Cytol. 2004;233:135-79.
Wang B, Hajano J-U-D, Ren Y, Lu C, Wang X. iTRAQ-based quantitative proteomics analysis of rice leaves infected by Rice stripe virus reveals several proteins involved in symptom formation. Virol J. 2015;12:99.

Wang RY, Li K. Host factors in the replication of positive-strand RNA viruses. Chang Gung Med J. 2012;35:111-24.

Yang WS, SriRamaratnam R, Welsch ME, Shimada K, Skouta R, Viswanathan VS, et al. Regulation of ferroptotic cancer cell death by GPX4. Cell. 2014;156:317-31.

Zeenko W, Ryabova LA, Spirin AS, Rothnie HM, Hess D, Browning KS, et al. Eukaryotic elongation factor 1A interacts with the upstream pseudoknot domain in the $3^{\prime}$ untranslated region of tobacco mosaic virus RNA. J Virol. 2002;76:5678-91.

Zhou Z, Han V, Han J. New components of the necroptotic pathway. Protein Cell. 2012:3:811-7.
Ready to submit your research? Choose BMC and benefit from:

- fast, convenient online submission

- thorough peer review by experienced researchers in your field

- rapid publication on acceptance

- support for research data, including large and complex data types

- gold Open Access which fosters wider collaboration and increased citations

- maximum visibility for your research: over $100 \mathrm{M}$ website views per year

At BMC, research is always in progress.

Learn more biomedcentral.com/submissions 